\title{
Predictive Graph Mining
}

\author{
Andreas Karwath and Luc De Raedt \\ Albert-Ludwigs-Universität Freiburg, Institut für Informatik, \\ Georges-Köhler-Allee 079, D-79110 Freiburg, Germany \\ \{karwath, deraedt\}@informatik. uni-freiburg.de
}

\begin{abstract}
Graph mining approaches are extremely popular and effective in molecular databases. The vast majority of these approaches first derive interesting, i.e. frequent, patterns and then use these as features to build predictive models. Rather than building these models in a two step indirect way, the SMIREP system introduced in this paper, derives predictive rule models from molecular data directly. SMIREP combines the SMILES and SMARTS representation languages that are popular in computational chemistry with the IREP rule-learning algorithm by Fürnkranz. Even though SMIREP is focused on SMILES, its principles are also applicable to graph mining problems in other domains. SMIREP is experimentally evaluated on two benchmark databases.
\end{abstract}

\section{Introduction}

In recent years, the problem of graph mining in general, and its application to chemical and biological problems, has become an active research area in the field of data-mining. The vast majority of graph mining approaches first derives interesting, i.e. frequent, patterns and then uses these as features to build predictive models. Several approaches have been suggested [1-8] for the task of identifying fragments which can be used to build such models. The earliest approaches to compute such fragments are based on techniques from inductive logic programming [1]. Whereas inductive logic programming techniques are theoretically appealing because of the use of expressive representation languages, they exhibit significant efficiency problems, which in turn implies that their application has been restricted to finding relatively small fragments in relatively small databases. Recently proposed approaches to mining frequent fragments in graphs such as gSpan [5], CloseGraph [9], FSG [2], and AGM [7] are able to mine complex subgraphs more efficiently. However, the key difficulty with the application of these techniques is - as for other frequent pattern mining approaches the number of patterns that are generated. Indeed, [6] report on $10^{6}$ of patterns being discovered. Furthermore, frequent fragments are not necessarily of interest to a molecular scientist. Therefore, [3] and [6] propose approaches that take into account the classes of the molecules. Kramer et al. compute all simple patterns that are frequent in the positives (or actives) and infrequent in the negatives (or inactives) [3]. Inokuchi et al. compute correlated patterns [6] . While Inokuchi et al. claim that the discovered patterns can be used for predictive purposes, they do not report on any predictive accuracies. 
The approach taken here is different: SMIREP produces predictive models (in the form of rule-sets) directly. SMIREP combines SMILES, a chemical representation language that allows the representation of complex graph structures as strings, with IREP $[10,11]$, a well-known rule-learner from the field of machine learning. SMIREP has been applied to two benchmark data sets (the mutagenicity dataset [12] and the AIDS Antiviral Screening Database [3]) and the experiments show that SMIREP produces small rule sets containing possibly complex fragments, that SMIREP is competitive in terms of predictive accuracy and that SMIREP is quite efficient as compared to other data mining systems.

Although, the SMIREP system is tailored towards the chemical domain, the approach can be employed as a general approach to build predictive models for graph mining domains.

The paper is organized as follows: in section 2 we give an overview of SMILES and SMARTS as language for chemical compounds and fragments as well as their applicability to other structured data, like non-directed graphs; section 3 gives an overview of the SMIREP system; in section 4, we report on experiments and findings conducted with SMIREP;finally, in section 5, we touch upon related work and conclude.

\section{SMILES and SMARTS}

SMILES (Simplified Molecular Input Line Entry System) [13] is a linear string representation language for chemical molecules. The SMILES language is commonly used in computational chemistry and is supported by most major software tools in the field, like the commercial Daylight toolkit or the Open-Source OpenBabel library.

The SMILES notations of chemical compounds are comprised of atoms, bonds, parathesis, and numbers:

- Atoms: Atoms are represented using their atomic symbols. E.g. C for carbon, $\mathrm{N}$ for nitrogen, or $\mathrm{S}$ for sulfur. For aromatic atoms, lower case letters are used, and upper case letters otherwise. Atoms with two letter symbols, like chlorine $(\mathrm{Cl})$ or bromine $(\mathrm{Br})$, are always written with the first letter in upper case and the second letter can be written upper or lower case. With a rare few exceptions, hydrogen atoms are not included in the string representation of a molecule.

- Bonds: Four basic bond types are used in the SMILES language: single, double, triple, and aromatic bonds, represented by the symbols: '-', '=', '\#', and ':' respectively. Single and aromatic bonds are usually omitted from SMILES strings. Not belonging to the four basic bonds are ionic bonds, or disconnections, represented by a '.'

- Branches: Branches are specified by enclosing brackets, "(" and ")", and indicate side-structures. A branch can, and often does, contain other branches.

- Cycles: Cyclic structures are represented by breaking one bond in each ring. The atoms adjacent to the bond obtain the same number. E.g. 'cccccc' denotes a (linear) sequence of six aromatic carbons and 'c1ccccc1' denotes 
a ring of six carbons. Here, we refer to the numbers indicating the cyclic structures of a compound as cyclic link numbers. These cyclic link numbers are not unique within the a SMILES representation of a molecule. E.g. 'c1cccc1Oc2cccc2' or 'c1cccc1Oc1cccc1' are valid notations for the same molecule.

To give an example, figure 1 shows the drug Guanfacine, which is used to treat high blood pressure (http://www.guanfacine.com/). It consist of one aromatic ring and several branches. This is reflected in its SMILES notation ' $\mathrm{c} 1(\mathrm{Cl}) \operatorname{cccc}(\mathrm{Cl})$ $\mathrm{c} 1 \mathrm{CC}(=\mathrm{O}) \mathrm{NC}(=\mathrm{N}) \mathrm{N}$ ', where the first substring reaching from the first ' $\mathrm{c} 1$ ' to the second ' $c 1$ ' code for the ring structure.

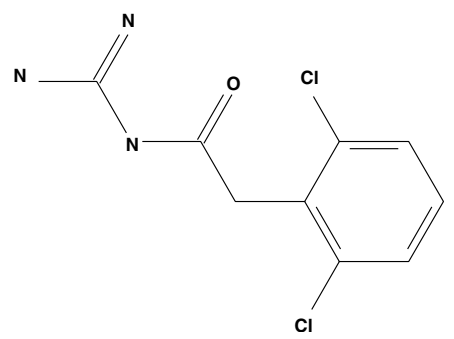

Fig. 1. A 2D graphical representation of Guanfacine. Its SMILES string representation is ' $\mathrm{c} 1(\mathrm{Cl}) \operatorname{cccc}(\mathrm{Cl}) \mathrm{c} 1 \mathrm{CC}(=\mathrm{O}) \mathrm{NC}(=\mathrm{N}) \mathrm{N}$ '.

The aim of the SMIREP system is to find predictive rules, or fragments, to differentiate active from non-active compounds. To represent such rules, we use the SMARTS language (http://www.daylight.com). SMARTS is a matching language for SMILES strings. While SMILES is a language representing molecules, SMARTS is a language representing fragments. In principle, SMARTS is derived from SMILES to represent (sub-)fragments. Almost all SMILES strings are valid SMARTS search strings. Furthermore, SMARTS allows the use of wildcards and more complicated constraints. Here, we use a subset of the SMARTS pattern language, that is, we use the SMILES notation for fragments and allow the atom wildcard '*' at the beginning of some matches. E.g. instead of a pattern ' $=\mathrm{O}$ ', the pattern ${ }^{*} *=\mathrm{O}^{\prime}$ is used.

Although, SMILES and SMARTS have been developed as representation languages for chemical compounds and fragments, the SMILES syntax is applicable to other undirected graphs. Vertices can be seen as atoms, while edges can be modeled by bonds. This allows the construction of arbitrary non-cyclic graphs like trees. Furthermore, using the notation of cyclic link numbers, more complex data can be modeled. An example is given in figure 2. The figure is modeled using only one type of vertices ('C') and one type of bond ('-'), but makes use of the cyclic link numbers. In the SMILES notation this would be expressed as 'C-12345C-6C-1C-2C-3C-4C-56' or 'C12345C6C1C2C3C4C56' for short.

This mapping between vertices and edges to atoms and bonds should, in principle, allow SMILES to be used for a wide range of other structured data, e.g. they could be used in general link mining. 


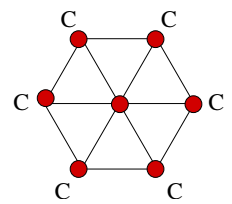

Fig. 2. The graphical representation of the graph 'C-12345C-6C-1C-2C-3C-4C-56', using a ' $\mathrm{C}$ ' for the vertices and and implicitly a '-' for edges.

\section{Algorithms}

In the following sections, the SMIREP system is explained in detail. SMIREP adopts ideas of the IREP algorithm [10,11], and tailors the original IREP to a predictive graph mining approach. The setting for SMIREP is similar as for IREP, the system learns predictive rules given a database of positive and negative examples. In detail, SMIREP grows rules for sets of randomly selected subsets of positive and negative examples, e.g. chemical compounds considered to be active or inactive. Rules are grown, using a randomly selected set of seeds and their corresponding SMILES representation as a starting point. The SMILES strings are decomposed into sub-fragments. All sub-fragments are stored in a labeled tree, the fragment tree. The leaf nodes of this tree, so called ground fragments (see below for details), are used to grow rules by either adding new ground fragments to a rule or refining sub-fragments in the rule according to the fragment tree. For a given seed, the best rule is selected according to the propositional version of FOIL's information gain measure [14] and reported back to the main SMIREP algorithm. The rules grown for all seeds are immediately pruned using a randomly selected pruning set, and the best rule of all pruned rules is selected and added to the rule set. The individual steps and terms are discussed in detail in the following sections.

\subsection{Fragment Decomposition}

The starting point for growing a rule are the ground fragments of a compounds SMILES string representation and its corresponding fragment tree.

As undirected graphs in general, and therefore SMILES strings in particular, have no unique representation, some canonical form is usually necessary to compare chemical compounds in graph notation. In SMIREP, the SMILES representation returned by the OpenBabel toolkit (http://openbabel.sourceforge.net) as the canonical form. This is only done once for each compound.

\section{$3.2 \quad$ Fragments}

For the decomposition of a SMILES representation, two different fragment types are of interest: linear and cyclic fragments. Linear fragments ( $L F s)$ are fragments defined by branches (or round brackets in the string), and cyclic fragments ( $C F s$ ) 
are defined by their numbered links within the SMILES representation of a molecule. Linear ground fragments $(L G F s)$, are the LFs of a molecule representation containing no branches. Cyclic ground fragments $(C G F s)$ are SMILES substring with a full cyclic structure. CGFs contain all atoms, bonds, redundant and non-redundant branching brackets), and all cyclic structure elements between the cyclic link numbers, including the corresponding atoms with their cyclic link numbers.

To give an example for both LGFs and CGFs, consider the SMILES representation string $S=$ 'c1c2c (ccc1) nc1c (cccc1c2Nc1ccc (cc1)S $(=\mathrm{O})$ )'. The set of LGFs for this SMILES representation is (in order of appearance from left to right): $\operatorname{LGFs}(\mathrm{S})=\{$ 'c1c2c', 'ccc1', 'nc1c', 'cccc1c2Nc1ccc', 'cc1', 'S', '=O' $\}$ and the corresponding set of CGFs is: $\operatorname{CGFs}(\mathrm{S})=\left\{{ }^{\prime} \mathrm{c} 1 \mathrm{c} 2 \mathrm{c}\left(\mathrm{ccc} 1\right.\right.$ ', ${ }^{\prime} \mathrm{c} 2 \mathrm{c}(\mathrm{ccc} 1) \mathrm{nc} 1 \mathrm{c}$ (cccc1c2', 'c1c(cccc1', 'c1ccc(cc1'\}. The corresponding 2D representation of this compound is given in 3 .

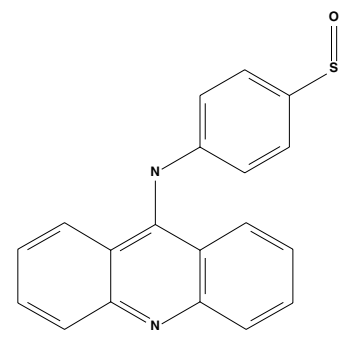

Fig. 3. Example 1: The 2D representation of the molecule having the SMILES code: 'c1c2c(ccc1)nc1c(cccc1c2Nc1ccc(cc1)S(=O))'.

\subsection{Decomposition}

For the decomposition of a SMILES string we have used an algorithm DECOMPOSE (not shown here). DECOMPOSE constructs a tree of (sub-)fragments of a SMILES representation of a molecule. It does so by repeatedly splitting substrings into linear and cyclic fragments, until no further splits are possible.

As input, the algorithm takes a SMILES string converted to the so-called internal SMILES representation. The internal representation assigns unique identifiers to all cyclic link numbers. This includes marking a cyclic link as either start (denoted by an ' $\{$ ')or end (denoted by a ' $\}$ '), and mapping a cyclic link number to a unique cyclic link number. This is necessary, as after decomposition, the fragments are re-combined by the system. Without assigning unique identifiers to the link numbers, the constructed SMARTS string for searching the database would produce wrong hits and therefore wrong results.

To simplify the extraction of CFs, the internal representation modifies the SMILES string regarding to the order of atoms and cyclic link numbers: it reverses all substrings containing an atom followed by cyclic link number at the beginning of a cyclic structure, e.g. $c c 1 c c c c 1$ to $c 1 c c c c c 1$. Given the original SMILES string: $S={ }^{\prime} c 1 c 2 c(\operatorname{ccc} 1) \operatorname{nc} 1 \mathrm{c}(\operatorname{cccc} 1 \mathrm{c} 2 \mathrm{Nc} 1 \mathrm{ccc}(\operatorname{cc} 1) \mathrm{S}(=\mathrm{O}))$ ', the internal 
representation would be: $S_{\text {int }}={ }^{\prime}{ }_{0} \mathrm{c}\left\{{ }_{1} \mathrm{cc}(\mathrm{ccc}\}_{0}\right) \mathrm{n}\left\{{ }_{2} \mathrm{cc}(\mathrm{cccc}\}_{2} \mathrm{c}\right\}_{1} \mathrm{~N}\left\{{ }_{3} \mathrm{cccc}(\mathrm{cc}\}_{3}\right) \mathrm{S}$ $(=\mathrm{O}))^{\prime}$, where $S_{\text {int }}$ denotes the string in internal representation. DECOMPOSE uses this internal representation and repeatedly splits substrings into linear and cyclic fragments, while building up the fragment tree. A node in the tree contains a SMILES substring, a link to its parent node, zero or more child nodes, and a label, indicating from which part of a linear or cyclic split the node originated.

Initially, all cyclic fragments of a SMILES string are found. This is done by simply parsing $S_{i n t}$ to find all strings $s$ of the form ' $\left\{{ }_{N} T\right\}_{N}$ ' where ' $\{$ ' and ' $\}$ ' are matching opening and closing brackets according to their unique identifier $N$, and $T$ is a string of symbols. For every matching substring $s$, a node $n_{s}$ is constructed containing a unique identifier as label, the substring $s$ as data, and no children. For this a method findCycles is used. All nodes are added to the root node as children. Similarly, the same is done to find linear fragments. The current SMILES string $S_{\text {int }}$ is matched against the pattern $A(B) C$ where $A, B$ and $C$ are substrings and '(' and ')' are corresponding brackets. E.g., consider the SMILES string ' $C C(=O C(O C) C) C C(=O) C$ '. The above method would split this into $A$ $=$ ' $C C$ ', $B=$ ' $=O C(O C) C$ ', and $C={ }^{\prime} C C(=O) C$ '. Similarly to findCycles, the method to find linear fragments is called findBranches. Both methods are called recursively on the found fragments, adding the new fragments as children to the previous level fragments, stopping if no more more CFs or LFs can be found.

To illustrate the basic decomposition performed by DECOMPOSE, consider the SMILES fragment representation $S={ }^{\prime} c 1 c 2 c(c c c 1) n c c(c c 2 N c c c c S(=O))$ ' or $\left.S_{\text {int }}={ }^{\prime}{ }_{0} c\left\{{ }_{1} c c(c c c\}_{0}\right) n\left\{{ }_{2} c c(c c c c\}_{2} c\right\}_{1} N c c c c S(=O)\right)$ '. The whole fragment tree constructed by DECOMPOSE is depicted in figure 4, and the 2D representation in figure 5 .

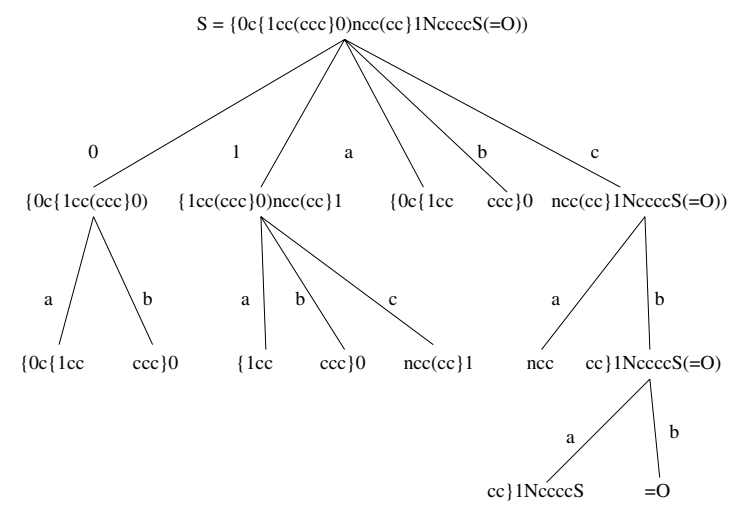

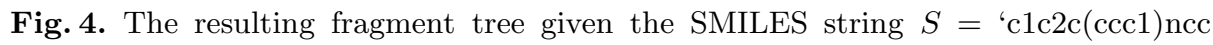
$(\operatorname{cc} 2 \operatorname{NccccS}(=\mathrm{O}))^{\prime}$. 


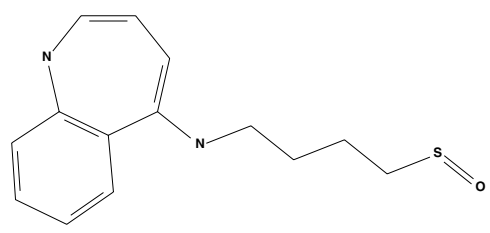

Fig. 5. Example 2. The 2D representation of the molecule having the SMILES code: 'c1c2c(ccc1) nc1c( $\operatorname{cccc} 1 \mathrm{c} 2 \mathrm{Nc} 1 \mathrm{ccc}(\operatorname{cc} 1) \mathrm{S}(=\mathrm{O}))$ '.

\subsection{SMIREP}

Like the original IREP algorithm [10], SMIREP employs a growing algorithm, GROW (see below), to grow rules according to a randomly selected set of examples (GrowPos and GrowNeg), and prunes the found rule immediately on a corresponding pruning example set (PrunePos and PrunNeg). After the pruning phase, SMIREP subsequently adds the pruned rule to a rule set. In the SMIREP system, the growing algorithm (GROW) specializes fragment strings in SMILES format using refinement operators. The refinement operators are described in detail later in this section. While in IREP, rules consist only of conjunctions of attribute-value pairs, in SMIREP rules consist of conjunction of SMARTS pattern strings. An example rule would be ' $\mathrm{NC}$ ' $\wedge$ ' $\mathrm{cc} 1 \mathrm{C}=\mathrm{Ccc} 1 \mathrm{cc}(\mathrm{cc})$ ', stating that pattern ' $\mathrm{NC}$ ' and pattern ' $\mathrm{cc} 1 \mathrm{C}=\mathrm{Ccc} 1 \mathrm{cc}(\mathrm{cc})$ ' have to occur in the compound simultaneously. As mentioned earlier, the SMIREP system uses a modified version of the original IREP algorithm, namely: rules are grown for a set of seeds only. $k$ seeds are selected randomly from the list of GrowPos. The default number of seeds used here was arbitrarily set to $k=10$.

The output of the SMIREP system, is a set of rules, predicting active compounds. A single rule in the rule set, consists of conjunctions of SMARTS pattern strings, their corresponding subfragments in the tree and their refinement history. The SMIREP algorithm is depicted as algorithm 1.

\subsection{The Refinement Operator}

The growing of rules in SMIREP is done using refinement operators, making the individual rule more specific. The refinement operator $r$ used here, refines a rule by using two different elementary refinements, Lengthening and Ascending. Both elementary refinement operators refine an existing rule by specializing it. The elementary refinements are:

- Lengthening: A ground fragment is added to the existing conjunctions of fragments.

- Ascending: The last fragment is refined with respect to its label and parent. Given the labels, the following patterns are constructed (according to their labels):

- $a$ : Construct patterns $A(B)$ and $A C$, with label $a b$ and $a c$ respectively.

- $b$ : Construct new pattern $A(B)$ with label $b a$. 


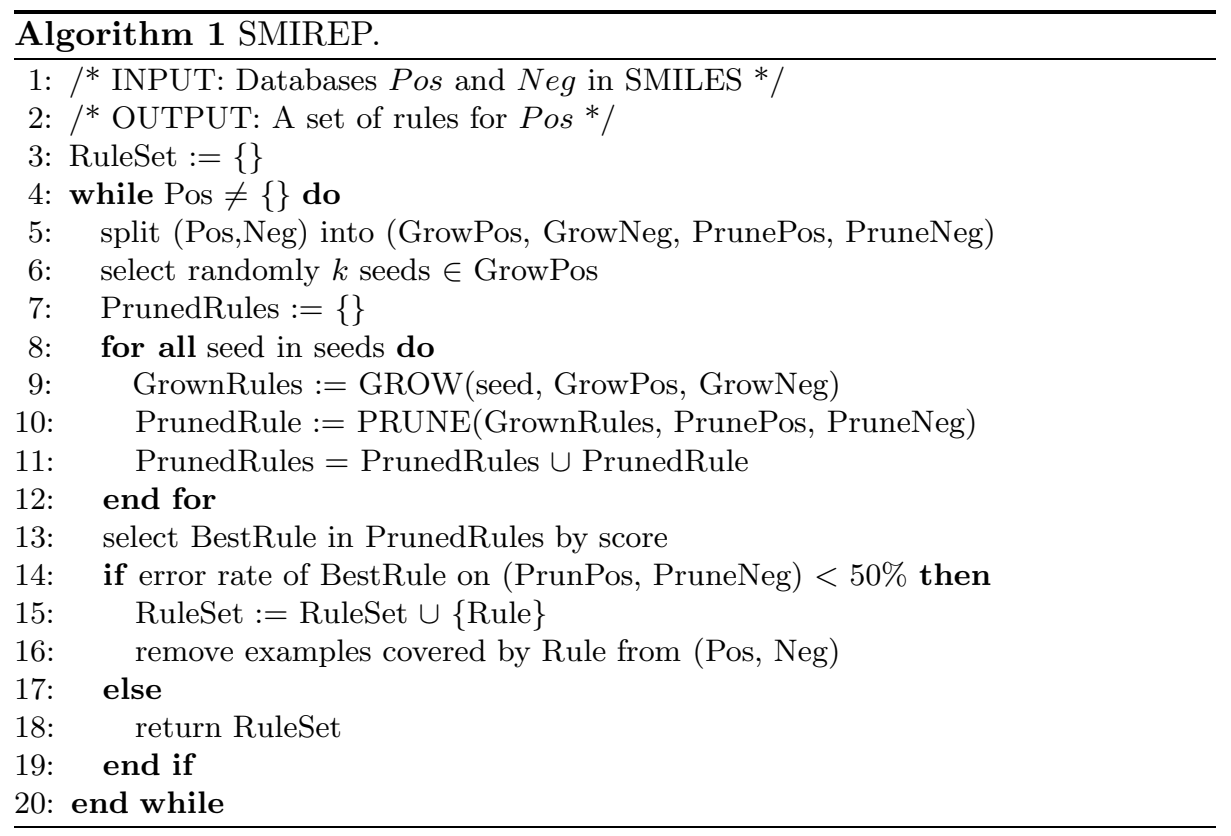

- $c$ : Construct new pattern $A C$ with label $a c$.

- $i$, where $i$ is an integer: Construct new pattern, where the pattern is the parent's pattern.

- $a b, a c, b a$, or $c a$ : Construct pattern $A(B) C$, where $A(B) C$ is the parent of $A, B$, or $C$.

- $r$, no construction of a new pattern, the fragment was a root node.

The Ascending elementary refinement only succeeds, if the refinement operator is able to construct a new pattern. This is not the case for patterns, or better fragments, representing the root node (labeled $r$ ). In these cases, the refinement operator fails and returns no new refinement. The refinement operator specializes a sub-fragment by either adding a new conjunction or refining an existing one, according to its label and parent. Please note, that in some cases the produced pattern can start with a bond. An additional atom wildcard is added to the pattern to transform it to a valid SMARTS search string. This refinement operator is used in the algorithm GROW.

Other graph mining approaches, like gSpan [5] or AprioriAGM [6] apply refinement operators resulting in minimal refinement steps. In contrast, the refinement operator used here, does not result in minimal refinements in graphs, it is rather comparable to macros within the ILP framework.

\subsection{Growing Rules}

The initial set of rules for growing, contains only the most general rule possible. For a SMARTS search, as well as the SMIREP system, this is the atom wildcard 
pattern ('*'). Rules are made more specific either by the Lengthening or by the Ascending operators. However, for the most general rule there is no possible Ascending refinement. Therefore, in the first step, the refinement operation returns all rules returned by the Lengthening operation, e.g. all rules containing a ground fragment. These rules become the current rule set. Each rule is scored according to a propositional version of the FOIL gain [14].

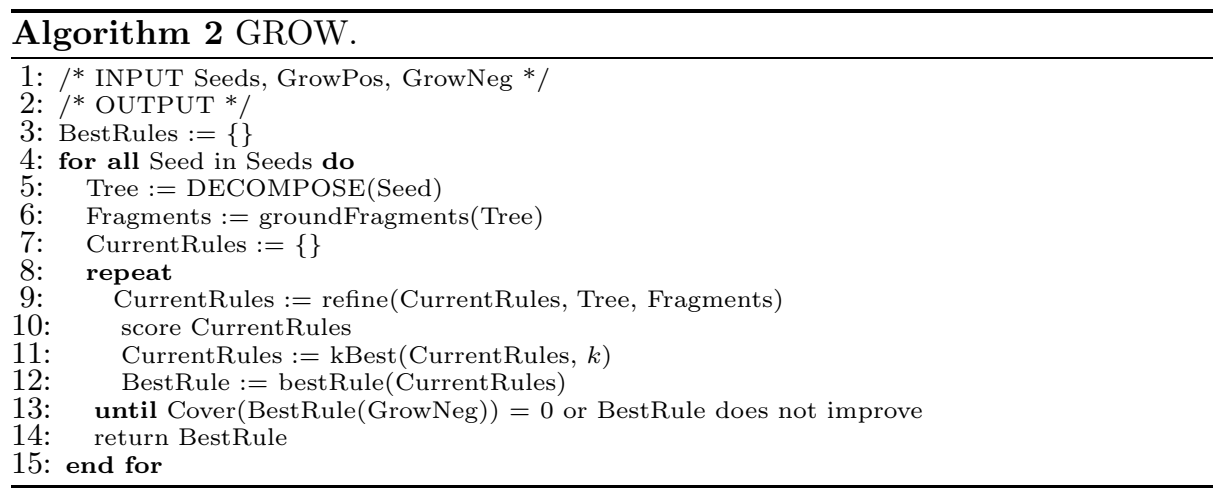

As refining all possible fragments of a molecule is computationally too expensive, only the k-best rules of the current rule set, are selected for further refinement, according to their score. Arbitrarily, $k$ has been set to 10 . The original growing algorithm in $[10,11]$ stops refining a rule, when no more negative examples are covered by the rule. In the work presented here, we have loosened this restrictive stopping condition for two reasons: First, it is theoretically possible, that a compound considered active is a subfragment of a compound considered to be inactive, resulting in an infinite loop in GROW. Second, as only the k-best scoring rules are selected for further refinement, the search for "better" patterns can get stuck in a local minimum, never reaching the "best" pattern, the initial seed fragment. In our implementation, GROW stops on refining a rule when either no negative examples are covered or the coverage of the current best rule concerning the negative examples does not decrease compared to the best rule from the previous refinement round. Please note, this is a conservative measure. The system does not consider potentially better rules, which might have occurred in later rounds.

\subsection{Pruning}

After growing a rule, the rule is immediately pruned. The pruning of a rule is performed by deleting any final sequence of refinements from a rule, and choosing the rule maximizing the following scoring function: $v^{*}$ (Rule, PrunePos, PruneNeg) $\equiv \frac{p-n}{p+n}$, where $p$ are the positive examples covered by the rule in the pruning set, and $n$ are the negative examples covered by the rule in the pruning set, as suggested by Cohen [11]. 
The deletion of final refinements is done in reverse to the original refinement: if the rule resulted from a lengthening operation, the final condition is deleted; if the rule resulted from a ascending operation, the last refinement is undone stepping further down the tree. The process is repeated until no more refinements can be be undone or one single fragment is left.

\subsection{Extension to SMIREP}

In many cases, additional information like numerical attributes is available about chemical compounds. We have modified our original SMIREP system to incorporate such extra information. The modification is done, by first discretising numerical attributes using equal frequency binning, using a default bin size of $s_{b i n}=10$, and then use the attribute-value information during the growing stage. For each seed, all bins are evaluated, resulting in attributes like ' $X \leq$ num' or ' $X>$ num', where $X$ is a numerical attribute and num a bin border. This information is used like a fragment during the growing stage. The new rule types produced by SMIREP are than a combination of SMILES fragments and intervals for numerical attributes.

\section{Implementation and Experiments}

The main SMIREP system has been developed in the programming language Python (version 2.2). Python allows rapid prototype development, due to a wide range of available libraries. The SMARTS matching has been done using the open-source chemical library OpenBabel (version 1.100, http://openbabel. sourceforge.net). All experiments were run on a PC running Suse Linux 8.2 with an Intel Pentium IV $-2.8 \mathrm{GHz}$ CPU and 2 GB of main memory.

\subsection{Mutagenesis Data}

The first experiment evaluates the SMIREP approach using the mutagenicity benchmark database $[12,15]$. The database was originally used to compare the ILP system PROGOL with attribute-value learners like linear regression based approaches. Here, we compare the results from SMIREP to the ones reported in the original paper.

The database contains structural information as well as chemical properties for a number of chemical compounds. These properties are $\epsilon_{L U M O}$ (Lowest unoccupied Molecule Orbital) and $\log P$ (Octanol-water partition coefficient), both are often used in QSAR studies. The database is divided into two sets: a regression friendly set with 188 entries and a regression unfriendly set with 42 entries. In the first experiment (SMIREP), only the structural information in form of SMILES strings was employed. The comparison of SMIREP to PROGOL can be seen in table 1. Although, PROGOL outperforms SMIREP on both test databases, we believe that SMIREP is still a valid approach, as no extensive background knowledge, like ILP's language bias nor chemical properties 
Table 1. The table shows the predictive accuracies of SMIREP compared to PROGOL (taken from [12]) on the two different datasets. The results on the regression friendly (188) dataset where calculated on a 10-fold cross validation. The accuracies reported on the regression unfriendly dataset (42) are based on leave one out validation.

\begin{tabular}{|l|c|c|}
\hline Method & 188 & 42 \\
\hline PROGOL+NS+S1 & 0.82 & 0.83 \\
PROGOL+NS+S2 & 0.88 & 0.83 \\
SMIREP & 0.78 & 0.81 \\
SMIREP+NS & 0.85 & 0.82 \\
\hline
\end{tabular}

were employed, still producing comparable results. In the second experiment $\left(\right.$ SMIREP + NS), the SMILES strings and numerical attributes $\left(\epsilon_{L U M O}\right.$ and $\log P$ ) where used together with the extended version of SMIREP. On the 188 dataset the accuracy of SMIREP improved, still not reaching the predictive accuracy of PROGOL + NS + S2, while nearly matching the predictive accuracy of both PROGOL experiments in the 42 test database.

\subsection{HIV Data}

For the second main experiment, we have used the DTP AIDS Antiviral Screening Database, originating from the NCI's development therapeutics program NCI/NIH (http://dtp.nci.nih.gov/). The database consists of syntactically correct SMILES representations of 41,768 chemical compounds [3]. Each data entry is classified as either active (CA), moderately active (CM), or inactive (CI). A total of 417 compounds are classified as active, 1,069 as moderately active, and 40,282 as inactive.

For the first experiment active-moderate, or AM for short, we have applied SMIREP to build a predictive models for the databases CA and CM in a ten-fold cross-validation. On average, it took 648 seconds to induce rules for each fold. The precision, recall, number of rules and predictive accuracy is given in table 2 . The default accuracy using a majority vote is $72.1 \%$. The produced rules vary for each fold, but often involve a rule containing the SMILES pattern ' $N=[N]=N$ '. This is not surprising, as this is an acidic Subfragment of Azidothymidine (AZT).

Table 2. The results of the active-moderate and active-inactive test for SMIREP. Training corresponds to the $90 \%$ training set and Testing to the $10 \%$ test set. The numbers in brackets indicate the standard deviation. The accuracy for the AI test is not reported (see text).

\begin{tabular}{|l|c|c|c|c|}
\hline & \multicolumn{2}{|c|}{ Active-Moderate(AM) } & \multicolumn{2}{c|}{ Active-Inactive(AI) } \\
& Training & Testing & Training & Testing \\
\hline Precision & $0.823(0.03)$ & $0.736(0.06)$ & $0.846(0.03)$ & $0.725(0.07)$ \\
Recall & $0.483(0.08)$ & $0.481(0.09)$ & $0.276(0.07)$ & $0.249(0.10)$ \\
Num Rules & $19.4(5.7)$ & $19.4(5.7)$ & $11.5(5.6)$ & $11.5(5.6)$ \\
Accuracy & & $0.80(0.04)$ & & N/A \\
\hline
\end{tabular}


In previous studies derivatives of AZT have already been identified as potential drug targets. Another interesting rule occurred in a number of folds, with pattern ' $\mathrm{Cc} 1 \mathrm{ccoc} 1 \mathrm{C}$ '. In the training set, 11 positive and no negative example are covered and in the test set five positive and no negative examples are covered. The pattern's 2D representation is depicted in figure 6 , pattern 1.

1.)

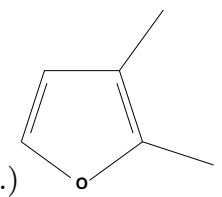

2.) $\mathrm{N}$

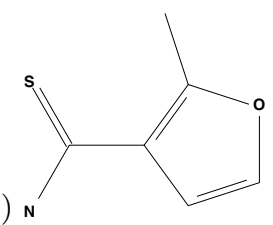

Fig. 6. A 2D graphical representation of an example rules found by SMIREP. Pattern 1 was found in the AM experiment. The rule contained only one single pattern ('Cc1ccoc1C'). Pattern 2 was found in AI experiment. The rule contained only one single pattern (' $\mathrm{NC}(=\mathrm{S}) \mathrm{c} 1 \mathrm{ccoc} 1 \mathrm{C}$ ').

The second experiment involving the HIV database was performed on the set of active against inactive chemical compounds using a 10-fold cross validation (AI experiment). On average, SMIREP took 7279 seconds, for each fold. In this experiment the average precision and recall on the test is lower to that obtained in the AM experiment. Here, we have not recorded the average prediction accuracy on the test set, as the default accuracy is $99.0 \%$ and a comparison would not result in any gain of information. One of the best rules found (pattern 2), was is a superstructure of the pattern 1 in figure 6 active-moderate experiment.

Interestingly, the recall decreases dramatically when compared to the AM experiment. We believe that this is due to the stopping criterion of a $50 \%$ error rate on the last best rule during the learning. In Fürnkranz work, two different stopping criteria where suggested: either a fixed cut of value of $50 \%$ error rate or the rule's accuracy is below the default accuracy. Here we have chosen the former, as using the default distribution would allow rules to be learned, having an error rate of up to $99 \%$.

Although, the reported average recall and precision of SMIREP in the AI experiment are relatively low, we believe that valuable patterns have been found. Further investigation is needed, to verify the effectiveness of SMIREP and the produced rules.

\section{Discussion and Conclusions}

Several approaches to mine graphs for predictive rules in the chemical domain have been proposed $[12,16,17,2]$. The SubdueCL system $[16,17]$ is able to induce predictive rules by graph-based relational learning (GBCL). SubdueCL is able to find Substructures in data, by extending candidate subgraphs by one edge searching for Substructures that best compress the graph given, using the MDL 
principle. The SubdueCL system has been applied to the PTE challenge data [17]. However, it has not been used to learn concepts for large databases like the HIV Database. Another approach, FSG [2], constructs features based on subgraph discovery and uses support vector machines (SVM) for the classification task. While in this work, a 5-fold cross validation was performed, the results seem to be comparable, slightly favoring the FSG approach. Still, we believe that SMIREP is a valid approach, as a very low number of rules are learned, enabling the examination of these rules by human experts. In $[18,19]$, the authors report on a system, DT-GBI, combining graph based induction of patterns with decision trees. The method constructs a decision tree, by pairwise chunking of GBI using the constructed patterns as attributes in the tree. The method employs a beam search to keep the computational complexity at a tolerant level. To some intent, SMIREP is similar to DT-GBI, by using a heuristic search as well as not inducing all possible frequent patterns. DT-GBI can be applied to a variety of graph structured data, not only to chemical compounds. DT-GBI was evaluated on a DNA dataset, therefore, no direct comparison to SMIREP are possible here. In future, this comparison has to be made.

As mentioned in section 2, the SMILES language can be used for other types of structured data. Hence we are convinced, that SMIREP can also be applied to other types of predictive graph mining, e.g. in the mining of messenger RNAs [20]. In a first step, a simple mapping between vertices and edges directly to atoms and bonds might allow the SMIREP system, using OpenBabel as matching library, to be used without alterations. However, this can only be done for problems having no more than four edge label types, or more vertice types than chemical atoms. For richer data structures, exceeding this limitations, a generic graph matching library would have to be employed.

The SMIREP system could be improved by using a different, more sophisticated, stopping condition during the learning stage. A further line of improvement lies in the upgrade from IREP to RIPPER [11] as underlying learning algorithm, repeatedly growing and pruning the found rules. Initial experiments on the mutagenesis database showed that the inclusion of numerical attributes, like $\epsilon_{L U M O}$ and $\log P$ can further improve the SMIREP system. Further experiments on other datasets are needed to the usefulness of the approach.

A novel approach to predictive graph mining was presented. SMIREP combines principles of the chemical representation language SMILES with the inductive rule learner IREP. As compared to contemporary approaches to graph mining, SMIREP directly builds a small set of predictive rules, whereas approaches such as gSpan, closeGraph [9], FSG [2], and AGM [7] typically find a large set of patterns satisfying a minimum frequency threshold, which are not necessarily predictive. Frequency based approaches attempt also to exhaustively search the space for all patterns that satisfy the frequency constraint. SMIREP in contrast - is a heuristic approach to find approximations of the most predictive rules. In this regard, SMIREP is more akin to the inductive logic programming approaches that induce rules from positive and negative examples, cf. [21]. On the one hand, these approaches are - in theory - more powerful because they 
employ Prolog, a programming language. On the other hand, they are - in practice - much more limited because expressive power comes at a computational cost. Indeed, molecular applications of inductive logic programming have so far not been able to induce rules containing large fragments, e.g. Warmr [1] only discovered rules of up to 7 literals in the PTE challenge [22] in a small data set. So, SMIREP tries to combine principles of inductive logic programming with those of graph mining.

Even though SMIREP was introduced in the context of molecular applications, it is clearly also applicable to general predictive graph mining problems. The authors hope that SMIREP will inspire further work on predictive graph mining.

\section{Acknowledgments}

This work has been partially supported by the EU IST project cInQ (Consortium on Inductive Querying). The authors would further like to thank Ross D. King for supplying the original mutagenesis dataset, and Christoph Helma and David P. Enot for helpful discussions and suggestions.

\section{References}

1. Dehaspe, L.: Frequent Pattern Discovery in First-Order Logic. K. U. Leuven (1998)

2. Deshpande, M., Kuramochi, M., Karypis, G.: Frequent sub-structure-based approaches for classifying chemical compounds. In: Proc. ICDM-03. (2003) 35-42

3. Kramer, S., De Raedt, L., Helma, C.: Molecular feature mining in HIV data. In Provost, F., Srikant, R., eds.: Proc. KDD-01, New York, ACM Press (2001) 136-143

4. Zaki, M.: Efficiently mining frequent trees in a forest. In Hand, D., Keim, D., Ng, R., eds.: Proc. KDD-02, New York, ACM Press (2002) 71-80

5. Yan, X., Han, J.: gspan: Graph-based substructure pattern mining. In: Proc. ICDM-02. (2002)

6. Inokuchi, A., Kashima, H.: Mining significant pairs of patterns from graph structures with class labels. In: Proc. ICDM-03. (2003) 83-90

7. Inokuchi, A., Washio, T., Motoda, H.: Complete mining of frequent patterns from graphs: Mining graph data. Machine Learning 50 (2003) 321-354

8. Kuramochi, M., Karypis, G.: Frequent subgraph discovery. In: Proc. ICDM-01. (2001) 179-186

9. Yan, X., Han, J.: Closegraph: Mining closed frequent graph patterns. In: Proc. KDD-03. (2003)

10. Fürnkranz, J., Widmer, G.: Incremental reduced error pruning. In Cohen, W.W., Hirsh, H., eds.: Proc. ICML 1994, Morgan Kaufmann (1994) 70-77

11. Cohen, W.W.: Fast effective rule induction. In: Proc. ICML 1995, Morgan Kaufmann (1995) 115-123

12. King, R.D., Muggleton, S., Srinivasan, A., Sternberg, M.J.E.: Structure-activity relationships derived by machine learning: The use of atoms and their bond connectivities to predict mutagenicity by inductive logic programming. Proc. of the National Academy of Sciences 93 (1996) 438-442 
13. Weininger, D.: SMILES, a chemical language and information system 1. Introduction and encoding rules. J. Chem. Inf. Comput. Sci. 28 (1988) 31-36

14. Quinlan, J.R.: Learning logical definitions from relations. Machine Learning 5 (1990) 239-266

15. Srinivasan, A., Muggleton, S., Sternberg, M.E., King, R.D.: Theories for mutagenicity: a study of first-order and feature based induction. A.I. Journal 85 (1996) 277-299

16. Cook, Holder: Graph-based data mining. ISTA: Intelligent Systems \& their applications 15 (2000)

17. Gonzalez, J.A., Holder, L.B., Cook, D.J.: Experimental comparison of graph-based relational concept learning with inductive logic programming systems. In: Proc. ILP 2003. Volume 2583 of Lecture Notes in Artificial Intelligence., Springer-Verlag (2003) 84-100

18. Warodom, G., Matsuda, T., Yoshida, T., Motoda, H., Washio, T.: Classifier construction by graph-based induction for graph-structured data. In: Advances in Knowledge Discovery and Data Mining. Volume 2637 of LNAI., Springer Verlag (2003) 52-62

19. Geamsakul, W., Matsuda, T., Yoshida, T., Motoda, H., Washio, T.: Constructing a decision tree for graph structured data. In: Proc. MGTS 2003, http://www.ar.sanken.osaka-u.ac.jp/MGTS-2003CFP.html (2003) 1-10

20. Horvath, T., Wrobel, S., Bohnebeck, U.: Relational instance-based learning with lists and terms. Machine Learning 43 (2001) 53-80

21. Muggleton, S.: Inverting entailment and Progol. In: Machine Intelligence. Volume 14. Oxford University Press (1995) 133-188

22. Srinivasan, A., King, R.D., Bristol, D.W.: An assessment of ILP-assisted models for toxicology and the PTE-3 experiment. In Džeroski, S., Flach, P., eds.: Proc. ILP-99. Volume 1634 of LNAI., Springer-Verlag (1999) 291-302 